N. Aoki

Nagoya Math. J.

Vol. 88 (1982), 155-160

\title{
HOMEOMORPHISMS WITHOUT THE PSEUDO-ORBIT TRACING PROPERTY
}

\author{
NOBUO AOKI
}

\section{§. Introduction}

Recently, A. Morimoto [5] proved that every isometry of a compact Riemannian manifold of positive dimension has not the pseudo-orbit tracing property, and that if a homeomorphism of a compact metric space has the pseudo-orbit tracing property then $E_{\varphi}=O_{\varphi}$ (see $\S 1$ for definition). The purpose of this paper is to show that every distal homeomorphism of a compact connected metric space has not the pseudo-orbit tracing property.

The author benefited from reading the papers by A. Morimoto [5, 6].

\section{§1. Definitions}

Let $\varphi: X \rightarrow X$ be a (self-) homeomorphism of a compact metric space $X$ with distance function $d$. A sequence of points $\left\{x_{i}\right\}_{i \in(a, b)}(-\infty \leq a<$ $b \leqslant \infty)$ is called a $\delta$-pseudo-orbit of $\varphi$ if $d\left(\varphi\left(x_{i}\right), x_{i+1}\right)<\delta$ for $i \in(a, b-1)$. A sequence $\left\{x_{i}\right\}$ is called to be $\varepsilon$-traced by $x \in X$ if $d\left(\varphi^{i}(x), x_{i}\right)<\varepsilon$ holds for $i \in(a, b)$. We say $(X, \varphi)$ to have the pseudo-orbit tracing property (abbrev. P.O.T.P.) if for every $\varepsilon>0$ there is $\delta>0$ such that every $\delta$ pseudo-orbit of $\varphi$ can be $\varepsilon$-traced by some point $x \in X$. The system $(X, \varphi)$ is said to be minimal if a $\varphi$-invariant closed set $K$ is necessarily $K=\emptyset$ or $K=X$. Let $A$ be a subset of the integer group $Z$. Then $A$ is syndetic if there is a finite subset $K$ of $Z$ with $Z=K+A$. Let $x \in X$. Then $x$ is an almost periodic point if $\left\{n \in Z: \varphi^{n}(x) \in U\right\}$ is a syndetic set for all neighborhoods $U$ of $x$. Let $(X, \varphi)$ be distal, that is, if $\inf _{n \in Z} d\left(\varphi^{n}(x), \varphi^{n}(y)\right)$ $=0$ then $x=y$. Then every $x \in X$ is an almost periodic point and the converse is true (p. 36 of [2]). It is clear that every equi-continuous homeomorphism has this property and is hence distal. But the converse does not hold. To check this for example, let $T^{2}$ be a 2 -dimensional torus

Received December 18, 1980. 
and define a homeomorphism $\varphi: T^{2} \rightarrow T^{2}$ by $\varphi\left(x_{1}, x_{2}\right)=\left(\alpha+x_{1}, n x_{1}+x_{2}\right)$ $\left(\left(x_{1}, x_{2}\right) \in T^{2}\right)$ where $\alpha \in T^{1}$ and $0 \neq n \in Z$. Then it will be easily checked that $\varphi$ is distal but not equi-continuous. A point $x \in X$ is said to be nonwandering (with respect to $\varphi$ ) if for every neighborhood $U$ of $x$, there is an $n>0$ with $U \cap \varphi^{n}(U) \neq \emptyset$. The set of all nonwandering points is called the nonwandering set and denoted by $\Omega(\varphi)$. Since $X$ is compact, we get $\Omega(\varphi) \neq \emptyset$. If in particular $(X, \varphi)$ is distal, then it is easily proved that $\Omega(\varphi)=X$ since every $x \in X$ is almost periodic. We know (cf. p. 132 of [7]) that there is always a Borel probability measure $\mu$ on $X$ which is preserved by $\varphi$ and $\varphi^{-1}$, and (cf. p. 135 of [7]) that if $(X, \sigma)$ is minimal then $\mu(U)>0$ for all non-empty open set $U$.

The set $2^{X}$ of all closed non-empty subsets of $X$ will be a compact metric space by the distance function $\bar{d}$ defined by

$$
\bar{d}(A, B)=\operatorname{Max}\left\{\operatorname{Max}_{b \in B} d(A, b), \operatorname{Max}_{a \in A} d(a, B)\right\} \quad\left(A, B \in 2^{X}\right)
$$

where $d(A, b)=\inf _{a \in A} d(a, b)$ (cf. p. 45 of [4]). We denote by $\operatorname{Orb}^{\delta}(\varphi)$ the set of all $\delta$-pseudo-orbits of $\varphi$ and by $\overline{\operatorname{Orb}^{\delta}(\varphi)}$ the set of all $A \in 2^{X}$, for which there is $\left\{x_{i}\right\} \in \operatorname{Orb}^{i}(\varphi)$ such that $A=\mathrm{Cl}\left\{x_{i}: i \in Z\right\}, \mathrm{Cl}$ denoting the closure. Let $E_{\varphi}$ denote the set of all $A \in 2^{X}$ such that for every $\varepsilon>0$ there is $A_{\varepsilon} \in \overline{\operatorname{Orb}^{\varepsilon}(\varphi)}$ with $\bar{d}\left(A, A_{\iota}\right)<\varepsilon$. An element $A$ of $E_{\varphi}$ is called an extended orbit of $\varphi$. On the other hand, we define $O_{\varphi}=\mathrm{Cl}\left\{O_{\varphi}(x): i \in Z\right\}$ where $O_{\varphi}(x)=\mathrm{Cl}\left\{\varphi^{i}(x): i \in Z\right\}$. We can easily see that $E_{\varphi}$ is closed in $2^{x}$ and that $O_{\varphi} \subset E_{\varphi}$ holds.

\section{§2. Results}

Throughout this section, $X$ will be a compact metric space with distance function $d$ and $\varphi$ will be a self-homeomorphism of $X$.

Theorem. Assume that $X$ is connected. If $(X, \varphi)$ is distal, then $(X, \varphi)$ has not P.O.T.P.

Lemma 1. If $(X, \varphi)$ has P.O.T.P., for every $\varepsilon>0$ and every $x_{0} \in \Omega(\varphi)$ there is a point $y \in X$ and an integer $k=k\left(x_{0}, \varepsilon\right)>0$ such that $O_{\varphi^{k}}(y) \subset$ $U_{s}\left(x_{0}\right)$.

Proof. Since $x_{0} \in \Omega(\varphi)$, for $\delta>0$ with $\delta<\varepsilon$ there are a point $x \in X$ and an integer $k>0$ such that $x$ and $\varphi^{k}(x)$ belong to $U_{\delta / 2}\left(x_{0}\right)$. Now, set $x_{n k+i}=\varphi^{i}(x)$ for $n \in \boldsymbol{Z}$ and $0 \leq i<k$. Obviously, $\left\{x_{i}\right\}_{i \in \boldsymbol{Z}}=\{\cdots, x, \varphi(x), \cdots$, $\left.\varphi^{k-1}(x), \cdots\right\} \in \operatorname{Orb}^{\delta}(\varphi)$. Hence we can find a point $y \in X$ such that $d\left(\varphi^{i}(y)\right.$, 
$\left.x_{i}\right)<\varepsilon$ for $i \in \boldsymbol{Z}$. In particular, $d\left(\varphi^{n k}(y), x_{n k}\right)<\varepsilon$ and hence $d\left(\varphi^{n k}(y), x\right)<\varepsilon$ for $n \in Z$. Therefore we have $O_{\varphi^{k}}(y) \subset U_{s}\left(x_{0}\right)$.

Corollary 1. Assume that $X$ is connected and not one point. If $(X, \varphi)$ is minimal, then $(X, \varphi)$ has not P.O.T.P.

Proof. Let $\varepsilon=$ diameter $(X) / 3$ and assume that $(X, \varphi)$ has P.O.T.P. By Lemma 1 we have that for some $x_{0} \in X$ there are $y \in X$ and $k>0$ with $O_{\varphi^{k}}(y) \subset U_{c}\left(x_{0}\right)$. Since $X$ is connected, $O_{\varphi^{k}}(y)=O_{\varphi}(y)=X$ and so diameter $(X) \leqslant 2 \varepsilon$. This is a contradiction.

Corollary 2. If $(X, \varphi)$ is minimal, then $E_{\varphi}=O_{\varphi}$.

Proof. It is proved by A. Morimoto that every $A \in E_{\varphi}$ is $\varphi$-invariant $(\varphi(A)=A)$. In fact, for every $\varepsilon>0$ there is $\varepsilon>\varepsilon_{1}>0$ such that $d(\varphi(x)$, $\varphi(y))<\varepsilon$ when $d(x, y)<\varepsilon_{1}$. By definition we can find $\left\{x_{i}\right\} \in \operatorname{Orb}^{\varepsilon_{1}}(\varphi)$ with $\bar{d}\left(A, \mathrm{Cl}\left\{x_{i}\right\}\right)<\varepsilon_{1}$. Set $y_{i}=\varphi\left(x_{i}\right)$ for $i \in Z$, then $d\left(y_{i}, x_{i+1}\right)<\varepsilon_{1}$ and so $\bar{d}\left(\mathrm{Cl}\left\{x_{i}\right\}, \mathrm{Cl}\left\{y_{i}\right\}\right)<\varepsilon_{1}$. It is clear that $d\left(\varphi\left(y_{i}\right), y_{i+1}\right)<\varepsilon$ for $i \in Z$. Hence, $\left\{y_{i}\right\} \in \operatorname{Orb}^{c}(\varphi)$. Let $A^{\prime}=\mathrm{Cl}\left\{x_{i}\right\}$. Then $\bar{d}\left(A^{\prime}, \varphi\left(A^{\prime}\right)\right)<\varepsilon_{1}$ and since $\bar{d}\left(A, A^{\prime}\right)$ $<\varepsilon_{1}$ we get $\bar{d}\left(\varphi(A), \varphi\left(A^{\prime}\right)\right)<\varepsilon$. Therefore

$$
\bar{d}(\varphi(A), A)<\bar{d}\left(\varphi(A), \varphi\left(A^{\prime}\right)\right)+\bar{d}\left(\varphi\left(A^{\prime}\right), A^{\prime}\right)+\bar{d}\left(A^{\prime}, A\right)<3 \varepsilon
$$

and so $\bar{d}(\varphi(A), A)=0$; i.e. $\varphi(A)=A$. Therefore we get $E_{\varphi}=\{X\}=O_{\varphi}$.

Lemma 2. If $(X, \varphi)$ has P.O.T.P., for every integer $k>0,\left(X, \varphi^{k}\right)$ has also P.O.T.P.

Proof. For every $\varepsilon>0$ there is $\delta>0$ such that $\left\{x_{i}\right\} \in \operatorname{Orb}^{\delta}(\varphi)$ is $\varepsilon$ traced by a point in $X$. Take $\left\{y_{i}\right\} \in \operatorname{Orb}^{\delta}(\varphi)$ and put $x_{n k+i}=\varphi^{i}\left(y_{n}\right)$ for $n$ $\in \boldsymbol{Z}$ and $0 \leqslant i \leqslant k-1$. Obviously, $\left\{x_{i}\right\} \in \operatorname{Orb}^{\delta}(\varphi)$. Hence there is $y \in X$ with $d\left(\varphi^{i}(y), x_{i}\right)<\varepsilon$ for $i \in Z$. In particular, $\left.d\left(\varphi^{k}\right)^{n}(y), y_{n}\right)=d\left(\varphi^{n k}(y), x_{n k}\right)$ $<\varepsilon$ for $n \in Z$. This completes the proof of Lemma 2 .

Lemma 3. Let $(X, \varphi)$ be distal. Then for every $x \in X,\left(O_{\varphi}(x), \varphi\right)$ is minimal.

Proof. Since every $x \in X$ is almost periodic under $\varphi$, for a neighborhood $U$ of $x$ there is a finite set $K=\left\{n_{1}, \cdots, n_{k}\right\}$ of $Z$ such that $Z=A$ $+K$ where $A=\left\{n \in Z: \varphi^{n}(x) \in U\right\}$. Hence $O_{\varphi}(x)=\mathrm{Cl}\left\{\varphi^{n}(x): n \in A\right\} \cup$ $\mathrm{Cl}\left\{\varphi^{n+n_{1}}(x): n \in A\right\} \cup \cdots \cup \mathrm{Cl}\left\{\varphi^{n+n_{k}}(x): n \in A\right\}$. Let $y \in O_{\varphi}(x)$. Then $O_{\varphi}(y)$ $\cap U \neq \emptyset$. This implies that $x \in O_{\varphi}(y)$. Hence $O_{\varphi}(x)=O_{\varphi}(y)$. 
Remark 1. If $(X, \varphi)$ is distal and topologically transitive, then it is clearly minimal (by Lemma 3 ).

We shall now give a proof of the theorem.

Assuming that $(X, \varphi)$ has P.O.T.P., we shall draw a contradiction. To do this, let $\varepsilon=$ diameter $(X) / 9$. Then there is $\delta>0$ with $\delta<\varepsilon$ such that every $\left\{z_{i}\right\} \in \operatorname{Orb}^{\delta}(\varphi)$ is $\varepsilon$-traced by a point of $X$. Lemma 1 insures us that for $y_{0} \in \Omega(\varphi)$ there are $y \in X$ and $k>0$ with $O_{\varphi^{k}}(y) \subset U_{s}\left(y_{0}\right)$. Put $\psi=\varphi^{k}$. Then $(X, \psi)$ has P.O.T.P. (by Lemma 2) and is distal. Since $X$ is connected and compact, we can take a sequence of points $\left\{p_{i}\right\}_{i=1}^{N}$ in $X$ such that $p_{1}$ $=y, d\left(p_{i}, p_{i+1}\right)<\delta / 2$ for $1 \leqslant i \leqslant N-1$ and such that $\bigcup_{i=1}^{N} U_{\delta}\left(p_{i}\right)=X$. Since $(X, \psi)$ is distal, every point of $X$ is almost periodic. Hence for $1 \leqslant$ $i \leqslant N$ there is an integer $c(i)>0$ such that $d\left(p_{i}, \psi^{c(i)}\left(p_{i}\right)\right)<\delta / 2$. Let us put

$$
\begin{array}{ll}
x_{i}=\psi^{-i}\left(p_{1}\right) & (i<0) \\
x_{i}=\psi^{i}\left(p_{1}\right) & (0 \leqslant i \leqslant c(1)-1) \\
x_{c(1)+i}=\psi^{i}\left(p_{2}\right) & (0 \leqslant i \leqslant c(2)-1) \\
\cdots & \\
x_{c(1)+\cdots+c(N-1)+i}=\psi^{i}\left(p_{N}\right) & (0 \leqslant i \leqslant c(N)-1) \\
x_{c(1)+\cdots+c(N)+i}=\psi^{i}\left(p_{N-1}\right) & (0 \leqslant i \leqslant c(N-1)-1) \\
\cdots & \\
x_{c(1)+2 c(2)+\cdots+2 c(N-1)+c(N)+i}=\psi^{i}\left(p_{1}\right) & (i \geqslant 0) .
\end{array}
$$

Obviously, $\left\{x_{i}\right\}_{i \in Z} \in \operatorname{Orb}^{\delta}(\psi)$ and $\bar{d}\left(\mathrm{Cl}\left\{x_{i}\right\}, X\right)<\delta$. By assumption, there is $z \in X$ with $d\left(\psi^{i}(z), x_{i}\right)<\varepsilon(i \in Z)$ so that $\bar{d}\left(O_{\psi}(z), X\right)<\delta+\varepsilon<2 \varepsilon$, and in particular

$$
\begin{array}{ll}
d\left(\psi^{i}(z), \psi^{i}\left(p_{1}\right)\right)=d\left(\psi^{i}(z), \psi^{i}(y)\right)<\varepsilon & (i<0), \\
d\left(\psi^{i+c}(z), \psi^{i}\left(p_{1}\right)\right)=d\left(\psi^{c+i}(z), \psi^{i}(y)\right)<\varepsilon & (i \geqslant 0)
\end{array}
$$

where $c=c(1)+c(N)+2 \sum_{i=2}^{N-1} c(i)$. This implies that

$$
\begin{array}{ll}
\psi^{i}(z) \in U_{\epsilon}\left(\psi^{i}(y)\right) \subset U_{\varepsilon}\left(O_{\psi}(y)\right) & (i<0), \\
\psi^{c+i}(z) \in U_{\varepsilon}\left(\psi^{i}(y)\right) \subset U_{\epsilon}\left(O_{\psi}(y)\right) & (i \geqslant 0)
\end{array}
$$

where $U_{\epsilon}\left(O_{\psi}(y)\right)=\bigcup_{h \in O_{\psi}(y)} U_{\iota}(h)$. Put $O_{\psi}^{-}(z)=\mathrm{Cl}\left\{\psi^{i}(z): i<0\right\}$ and $O_{\psi}^{+}(z)$ $=\mathrm{Cl}\left\{\psi^{i}(z): i \geqslant 0\right\}$. Then we have that $O_{\psi}^{-}(z) \subset U_{\epsilon}\left(O_{\psi}(y)\right)$ and $\psi^{c} O_{\psi}^{+}(z) \subset$ $U_{\varepsilon}\left(O_{\psi}(y)\right)$. Since $O_{\psi}^{-}(z) \cup O_{\psi}^{+}(z)=O_{\psi}(z)$, by Baire's theorem either $O_{\psi}^{-}(z)$ or $O_{\psi}^{+}(z)$ has non-empty interior in the set $O_{\psi}(z)$.

Let $\mu$ be a $\psi$-invariant Borel probability measure of $O_{\psi}(z)$. Since 
$\left(O_{\psi}(z), \psi\right)$ is minimal by Lemma 3, every non-empty open set in $O_{\psi}(z)$ has $\mu$-positive measure. When the interior of $O_{\psi}^{-}(z)$ in $O_{\psi}(z)$ is non-empty, it is easy to see that $O_{\psi}^{-}(z)=\psi O_{\psi}^{-}(z)$ and so $O_{\psi}^{-}(z)=O_{\psi}(z)$. Indeed, assume $\psi^{-1} O_{\psi}^{-}(z) \subseteq O_{\psi}^{-}(z)$. Then $V=\bigcap_{k \geqslant 0} \psi^{-k} O_{\psi}^{-}(z)$ does not contain the interior of $O_{\psi}^{-}(z)$ in $O_{\psi}(z)$. Hence $\mu\left(O_{\psi}^{-}(z) \backslash V\right)>0$. Since $O_{\psi}^{-}(z)=\bigcup_{k>0} \psi^{-k}\left\{O_{\psi}^{-}(z) \backslash\right.$ $\left.\psi^{-1} O_{\psi}^{-}(z)\right\} \cup V$, we get $\mu\left(O_{\psi}^{-}(z) \backslash \psi^{-1} O_{\psi}^{-}(z)\right)>0$, thus contradicting $\mu\left(O_{\psi}^{-}(z)\right) \leqslant$ 1. If the interior of $O_{\psi}^{+}(z)$ in $O_{\psi}(z)$ is non-empty; i.e. $\mu\left(O_{\psi}^{+}(z)\right)>0$, then it follows that $O_{\psi}^{+}(z)=O_{\psi}(z)$. Obviously $O_{\psi}(z)=\psi^{c} O_{\psi}^{+}(z)$. In any case we get $O_{\psi}(z) \subset U_{\varepsilon}\left(O_{\psi}(y)\right)$ so that $O_{\psi}(z) \subset U_{s}\left(O_{\psi}(y)\right) \subset U_{2 \varepsilon}\left(y_{0}\right)$ (because $O_{\psi}(y)$ $\left.\subset U_{\varepsilon}\left(y_{0}\right)\right)$. Since $2 \varepsilon>\bar{d}\left(O_{\psi}(z), X\right)=\max _{x \in X} d\left(O_{\psi}(z), x\right)$, we have $X=$ $U_{2 \varepsilon}\left(O_{\psi}(z)\right)$ from which $X=U_{4 \varepsilon}\left(y_{0}\right)$; i.e. diameter $(X) \leqslant 8 \varepsilon$. This is a contradiction.

Remark 2. We know (Application 2 of [1]) that every (group) automorphism $\sigma$ of a zero-dimensional compact metric group $X$ has P.O.T.P. If $(X, \sigma)$ has zero topological entropy (the existence of such automorphisms is known), then we can prove (cf. Lemma 14 of [1]) that $X$ contains a sequence $X=X_{0} \supset X_{1} \supset \cdots$ of completely $\sigma$-invariant normal subgroups such that $\cap X_{n}$ is trivial and for every $n \geqslant 0, X_{n} / X_{n+1}$ is a finite group. Hence for $x, y \in X(x \neq y)$ there is $n>0$ such that $x y^{-1} \notin X_{n}$. Since $\sigma^{j}\left(X_{n}\right)$ $=X_{n}$ for all $j \in Z$, we get easily $\sigma^{\jmath}\left(x y^{-1}\right) \notin X_{n}(j \in Z)$, which implies that $d\left(\sigma^{j}(x), \sigma^{j}(y)\right) \geqslant d\left(\sigma^{j}(x) X_{n}, \sigma^{j}(y) X_{n}\right)>0$ (the distance function $d$ is a translation invariant metric of $X)$. Since $X_{n} / X_{n+1}$ is a finite group, we get $\inf _{j} d\left(\sigma^{j}(x), \sigma^{j}(y)\right)>0$; i.e. $(X, \sigma)$ is distal. Therefore every zero-dimensional automorphism with zero topological entropy is distal and has P.O.T.P. This shows that the assumption of connectedness in the theorem can not drop out.

\section{REFERENCES}

[1] N. Aoki, The splitting of zero-dimensional automorphisms and its application, (to appear in Collq. Math.)

[2] R. Ellis, Lectures on Topological Dynamics, Benjamin, New York 1969.

[3] R. Bowen, $\omega$-limit sets for Axiom A diffeomorphisms, J. Diff. Eq., I8 (1975), 333-339.

[4] C. Kuratowskii, Topologie, Vol. II, Warzawa, 1961.

[5] A. Morimoto, Stochastically stable diffeomorphisms and Takens conjecture, (preprint)

[6] - Stochastic stability of group automorphisms, (preprint)

[ 7 ] P. Walters, Ergodic Theory-Introductory Lectures, Springer Lec. notes, No. 458, 1975. 
Department of Mathematics Tokyo Metropolitan University Fukazawa, Setagaya-ku

Tokyo 158, Japan 